\title{
Beyond Alice and Bob: Improved Inapproximability for Maximum Independent Set in CONGEST
}

\author{
Yuval Efron \\ Technion \\ efronyuv@gmail.com
}

\author{
Ofer Grossman \\ MIT \\ ofer.grossman@gmail.com
}

\author{
Seri Khoury \\ UC Berkeley \\ seri_khoury@berkeley.edu
}

\begin{abstract}
By far the most fruitful technique for showing lower bounds for the CONGEST model is reductions to two-party communication complexity. This technique has yielded nearly tight results for various fundamental problems such as distance computations, minimum spanning tree, minimum vertex cover, and more.

In this work, we take this technique a step further, and we introduce a framework of reductions to $t$-party communication complexity, for every $t \geq 2$. Our framework enables us to show improved hardness results for maximum independent set. Recently, Bachrach et al. [PODC 2019] used the two-party framework to show hardness of approximation to maximum independent set. They show that finding a $(5 / 6+\epsilon)$-approximation requires $\Omega\left(n / \log ^{6} n\right)$ rounds, and finding a $(7 / 8+\epsilon)$-approximation requires $\Omega\left(n^{2} / \log ^{7} n\right)$ rounds, in the CONGEST model where $n$ is the number of nodes in the network.

We improve the results of Bachrach et al. by using reductions to multi-party communication complexity. Our results:

(1) Any algorithm that finds a $(1 / 2+\epsilon)$-approximation to maximum independent set in the CONGEST model requires $\Omega\left(n / \log ^{3} n\right)$ rounds.

(2) Any algorithm that finds a $(3 / 4+\epsilon)$-approximation to maximum independent set in the CONGEST model requires $\Omega\left(n^{2} / \log ^{3} n\right)$ rounds.
\end{abstract}

ACM Reference Format:

Yuval Efron, Ofer Grossman, and Seri Khoury. 2020. Beyond Alice and Bob: Improved Inapproximability for Maximum Independent Set in CONGEST. In ACM Symposium on Principles of Distributed Computing (PODC '20), August 3-7, 2020, Virtual Event, Italy. ACM, New York, NY, USA, 10 pages. https://doi.org/10.1145/3382734.3405702

\section{INTRODUCTION AND RELATED WORK}

Consider a network of $n$ nodes, where each has a unique $O(\log n)$ bit identifier, and they can communicate with each other via synchronized communication rounds. In each round, each node can send a (possibly different) $O(\log n)$-bit message to each of its neighbors. The task of the nodes is to compute some function of the network (e.g., its diameter, the value of a maximum independent set, etc.), while minimizing the number of communication rounds.

Permission to make digital or hard copies of all or part of this work for personal or classroom use is granted without fee provided that copies are not made or distributed for profit or commercial advantage and that copies bear this notice and the full citation on the first page. Copyrights for components of this work owned by others than ACM must be honored. Abstracting with credit is permitted. To copy otherwise, or republish, to post on servers or to redistribute to lists, requires prior specific permission and/or a fee. Request permissions from permissions@acm.org.

PODC '20, August 3-7, 2020, Virtual Event, Italy

(C) 2020 Association for Computing Machinery.

ACM ISBN 978-1-4503-7582-5/20/08 . .\$15.00

https://doi.org/10.1145/3382734.3405702
This model is well known as the CONGEST model, and it is one of the major models of theoretical distributed graph algorithms [22].

In recent years, our understanding of the complexity of some problems in the CONGEST model has been substantially improving, thanks to a fruitful technique for proving lower bounds via reductions to two-party communication complexity. This technique, that was implicitly introduced by Peleg and Rubinovich [23, 24], and explicitly and formally defined by Das Sarma et al. [27, 28], was used to prove many lower bounds for fundamental graph problems, such as distance computations [1, 15, 18], minimum spanning tree [13, 23, 27], minimum cut [16, 27], minimum vertex cover [8], constructing and verifying spanners [6, 7], subgraph detection [11, 14, 17], approximate max-clique [10], hardness of distributed optimization [4], distributed random walks [21], and more (a complete list of papers is infeasible).

While the two-party communication complexity model is already a very successful source for reductions, a natural question is whether using more players can bring us even closer to a satisfactory understanding of the CONGEST model. In [11], the authors use a reduction to the three-party set-disjointness problem to show a lower bound for triangle detection in the CONGEST-Broadcast model, where, unlike in the CONGEST model, the nodes are not allowed to send different messages to different neighbors in each round, and they can only broadcast one $O(\log n)$-bit message to all their neighbors in each round. This lower bound doesn't translate to the CONGEST model (In fact, no lower bound better than 1 round is known for triangle detection in the CONGEST model $[2,14]$ ). Whether multi-party communication complexity is of any use to show stronger results for the CONGEST model has remained open.

In this work, we introduce a framework of reductions to multiparty communication complexity. Our framework enables us to show improved hardness results to maximum independent set in the CONGEST model. Recently, Bachrach et al.[4] showed that any algorithm that finds a $(5 / 6+\epsilon)$-approximation to maximum independent set must spend $\Omega\left(n / \log ^{6} n\right)$ rounds. Furthermore, they showed that finding a $(7 / 8+\epsilon)$-approximation requires $\Omega\left(n^{2} / \log ^{7} n\right)$ rounds, which is nearly tight, as any problem can be solved in $O\left(n^{2}\right)$ rounds in the CONGEST model. Our results:

THEOREM 1. For any constant $0<\epsilon<1 / 2$, any algorithm that finds $a(1 / 2+\epsilon)$-approximation to maximum independent set in the CONGEST model requires $\Omega\left(n / \log ^{3} n\right)$ rounds.

THEOREM 2. For any constant $0<\epsilon<1 / 4$, any algorithm that finds $a(3 / 4+\epsilon)$-approximation to maximum independent set in the CONGEST model requires $\Omega\left(n^{2} / \log ^{3} n\right)$ rounds.

While our results are not necessarily tight, we hope that our technique could pave the way for more and stronger lower bounds in the CONGEST model. One important property of our technique 
is that it doesn't suffer from the same limitations as the two-party framework, on which we elaborate later. We note our results hold even against randomized algorithms that succeed with probability $p \geq 2 / 3$, and even for constant diameter graphs. The hard instances that are used to prove Theorems 1 and 2 are weighted graphs, but we can extend our arguments for unweighted graphs as well by losing a logarithmic factor in the lower bounds (in terms of the number of rounds), as explained in Remark 1.

To prove Theorems 1 and 2 we use reductions to $t$-party communication complexity where we use $t=O(1 / \epsilon)$ players. For $t=2$, our constructions are similar to the ones presented by [4], and can be viewed as simplified versions of them. While we also get a minor improvement in terms of the number of rounds (and not only in terms of approximations) it is worth pointing out that the improvements we get in terms of the number of rounds are not artifacts of the multi-party construction, but rather are artifacts of the simplifications compared to [4].

The two-party reduction technique in a nutshell: The vast majority of these reductions rely on the high communication complexity of the two-party set-disjointness problem. In the set-disjointness problem, there are two players, Alice and Bob, who receive two input strings $x, y \in\{0,1\}^{k}$, where Alice receives $x$, Bob receives $y$, and they wish to know if their strings intersect. That is, they wish to know if there is an index $i \in[k]$, such that $x_{i}=y_{i}=1$. It is well known that Alice and Bob must exchange $\Omega(k)$ bits in order to solve set-disjointness $[20,26]$. A reduction to the set-disjointness problem is usually applied in the following manner. Assume that we want to prove a lower bound in the CONGEST model for deciding whether an input graph has some property $P$ (for example, $P$ can be the property of having a diameter less than 5). Alice and Bob, before they start the protocol for set-disjointness, decide on a fixed graph construction $G=(V, E)$, and a partition of $G$ into two graphs $G_{A}=\left(V_{A}, E_{A}\right)$ and $G_{B}=\left(V_{B}, E_{B}\right)$, where $G_{A}$ is owned by Alice, and $G_{B}$ is owned by Bob. Then, each of the players adds some edges to their own graph based on their input string, where Alice adds edges based on $x$, Bob adds edges based on $y$, and the set of cut edges between $G_{A}$ and $G_{B}$ is fixed and doesn't depend on the input strings. The construction is defined in a way such that the strings $x$ and $y$ are disjoint if and only if the graph, with the additional edges based on the input strings, has the property $P$. Hence, in order to find whether $x$ and $y$ are disjoint, Alice and Bob can simulate a CONGEST algorithm that checks whether an input graph has the property $P$. Simulating the CONGEST algorithm in the two-party communication complexity model is done as follows. For messages that are sent on edges in $G_{A}$, Alice can simulate these messages without any communication with Bob. Similarly, Bob can simulate the messages that are sent on edges in $G_{B}$, without any communication with Alice. For the other messages, the ones that are sent on edges in the cut between $G_{A}$ and $G_{B}$, Alice and Bob exchange messages: if there is a message from a node in $G_{A}$ to a node in $G_{B}$, then Alice sends this message to Bob, and vice-versa. The conclusion that is made in such reductions is that if there is an $r$-round algorithm for deciding $P$ in the CONGEST model, and if the number of edges on the cut betwen $G_{A}$ and $G_{B}$ is at most $c$, then there is a protocol for solving two-party set-disjointness that uses $O(r \cdot c \cdot \log n)$ bits. This is because on each edge on the cut, the CONGEST algorithm sends $O(\log n)$ bits in each of the $r$ rounds. Hence, since the communication complexity of set-disjointness is $\Omega(k)$, we get a lower bound of $r=\Omega\left(\frac{k}{c \log n}\right)$ rounds for any algorithm for deciding $P$ in the CONGEST model. That is, the smaller the cut, the stronger the lower bound.

Censor-Hillel at al.[8] showed a small-cut two-party construction to prove a lower bound for maximum independent set, where they show that any algorithm for finding or computing the optimal value of a maximum independent set must spend $\Omega\left(n^{2} / \log ^{2} n\right)$ rounds. An independent set in a graph is a subset of the nodes where no two nodes in the subset are neighbors. A maximum independent set in a (possibly weighted) graph is an independent set of maximum total weight, where by total we mean the sum of weights of nodes in the independent set. Independent sets play vital role in theoretical and practical computer science, and the problem of computing exact or approximate maximum independent set has been attracting attention recently in the CONGEST model $[4,5,8,19]$. However, in terms of upper bounds, we are still unable to find fast algorithms that achieve approximation factors better than $\Delta$, where $\Delta$ is the maximum degree of a node in the graph. If one is happy with $\Delta$ approximation, or $(1+\epsilon) \Delta$-approximation, then very fast and even sub-logarithmic algorithms exist $[5,19]$. In terms of lower bounds, recently Bachrach et al. [4] built on the small-cut construction of [8], together with a very clever use of error-correcting codes, to prove a near-linear hardness for $(5 / 6+\epsilon)$-approximation, and near-quadratic one for $(7 / 8+\epsilon)$-approximation.

Limitations of the two-party framework: As pointed out by [4], the two-party framework suffers from some limitations. Especially, but not only, when trying to use it to prove hardness of approximation. For example, for the maximum independent set problem, the two-party framework cannot show any lower bound against algorithms that achieve (1/2)-approximation. This is because Alice and Bob can compute the optimal solutions for maximum independent set in the graphs $G_{A}$ and $G_{B}$, without any communication, where Alice finds the optimal solution in $G_{A}$, and Bob finds the optimal solution in $G_{B}$. The maximum of the two values is always at least half of the optimal solution for $G$. Hence, by just exchanging the two values, which takes $O(\log n)$ bits of communication, Alice and Bob can find a (1/2)-approximation to maximum independent set. Since $O(\log n)$ bits can be sent in one round in the CONGEST model, no lower bound for this approximation factor can be shown by using the two-party framework. Similarly, the two party framework suffers from a limitation when trying to show a lower bound for (3/2)-approximation to minimum vertex cover, where the argument for vertex-cover is not trivial and was proved also in [4].

By using more players, the framework doesn't suffer from the same limitations as in the two-party case. For example, with respect to approximating maximum independent set, the argument above translates only to a limitation of showing a $(1 / t)$-approximation. Hence, the more players we use, the less restrictive the limitations we get.

The Challenge: Perhaps the first attempt that one would try in 
order to extend the two-party framework to the multi-party case is to use a reduction to the multi-party set-disjointness problem. In the multi-party set disjointness problem, there are $t$ players $p_{1}, \cdots, p_{t}$ Each receives a string $x^{i} \in\{0,1\}^{k}$, and they wish to know if the strings all intersect on the same index. That is, they wish to know if there is an index $m \in[k]^{1}$ satisfying $x_{m}^{1}=x_{m}^{2}=\cdots=x_{m}^{t}=$ 1. However, using a reduction to the multi-party set-disjointness problem is not a simple task, and as $t$ gets larger, the task becomes more challenging. This is because in the non-intersecting case, there are many sub-cases of pairwise intersections, and the reduction needs to take into account all these sub-cases. For example, if we try to extend the reduction of [4] to the multi-party set-disjointness problem, in the non-intersecting case, for every pair $i \neq j \in[t]$, whether the strings $x^{i}$ and $x^{j}$ are intersecting or not influences the size (or weight) of the maximum independent set. Hence, for the non-intersecting case, the reduction needs to take into account all the sub-cases of pairwise intersections, and, the more players we have, the more sub-cases we get, and the more infeasible the reduction becomes.

In order to overcome this challenge, we use reductions to a certain promise pairwise disjointness problem, rather than the multiparty set-disjointness problem. In this promise pairwise disjointness problem, there are $t$ players each receiving a string $x^{i} \in\{0,1\}^{k}$, with the promise that the strings are either all intersecting in the same index, or pairwise disjoint. That is, in the non-intersecting case, for all pairs $i \neq j \in[t]$, it holds that $x^{i}$ and $x^{j}$ are disjoint. Most importantly, we don't have many sub-cases of pairwise intersections in the non-intersecting case. The communication complexity of this promise pairwise disjointness problem is $\Omega(k / t \log t)$ [9], which is large enough for our needs, and we are able to use it to prove our results.

Road-map: In Section 2, we begin with some useful definitions and tools. In Section 3, we present our framework of reductions to the multi-party communication complexity model. The technical heart of the paper is provided in Sections 4 and 5, where we show our linear and quadratic lower bounds, respectively.

\section{PRELIMINARIES}

\subsection{Multi-party Communication Complexity}

Our lower bounds rely on reductions to the number-in-hand model of multi-party communication complexity. In the number-in-hand model, there are $t$ players, each is holding an input $x^{i} \in\{0,1\}^{k}$, and they wish to compute a joint function of their inputs $f\left(x^{1}, \cdots, x^{t}\right)$, where $t$ and $k$ are parameters of the model. The communication setting in the number-in-hand model can be defined in various ways. In this work we use the shared blackboard model (see also, for example, [25]), where the players can exchange messages by writing them on a shared blackboard that is visible to all the players. The communication complexity in this model is formally defined as follows.

DEFINITION 1. [Communication Complexity-Shared Blackboard]

\footnotetext{
${ }^{1}$ Throughout the paper, for any positive integer $k$, we denote by $[k]$ the set of positive integers $\{1,2, \cdots, k\}$.
}

Let $k \geq 1, t \geq 2$ be two integers, $f$ be a (possibly partial) Boolean function $f: \prod_{i=1}^{t}\{0,1\}^{k} \rightarrow\{$ TRUE, FALSE $\}$, and $Q$ be the family of protocols that compute $f$ correctly with probability at least $2 / 3$, in the shared blackboard model. Given $t$ inputs $x^{1}, \cdots, x^{t}$, denote by $\pi_{Q}\left(x^{1}, \cdots, x^{t}\right)$ the transcript of a protocol $Q$ on the inputs $x^{1}, \cdots, x^{t}$, i.e. the sequence of bits that are written on the shared blackboard by all participants during execution of $Q$. The cost of a protocol $Q$ is

$$
\operatorname{Cost}(Q)=\max _{x^{1}, \cdots, x^{t} \in\{0,1\}^{k}}\left|\pi_{Q}\left(x^{1}, \cdots, x^{t}\right)\right|
$$

The communication complexity of $f$, denoted by $C C_{f}(k, t)$, is defined to be the minimum cost over all the possible protocols that compute $f$ correctly with probability at least $2 / 3$ :

$$
C C_{f}(k, t)=\min _{Q \in Q} \operatorname{Cost}(Q)
$$

Our lower bounds for the CONGEST model are achieved via reductions to the promise pairwise disjointness function. For two strings $x, y \in\{0,1\}^{k}$, we say that $x$ and $y$ are disjoint if $\sum_{j=1}^{k} x_{j} y_{j}=$ 0 .

Definition 2. [Promise Pairwise Disjointness]

Let $k \geq 1, t \geq 2$, and $x^{1}, \cdots, x^{t} \in\{0,1\}^{k}$, with the promise that the strings $x^{1}, \cdots, x^{t}$ are either uniquely intersecting, or pairwise disjoint. That is, either there is an $m \in[k]$ satisfying $x_{m}^{1}=x_{m}^{2}=$ $\cdots=x_{m}^{t}=1$, or $x^{i}$ and $x^{j}$ are disjoint for all pairs $i \neq j \in[t]$. The promise pairwise disjointness function outputs TRUE if the strings are pairwise disjoint, and FALSE if they are uniquely intersecting.

Chakrabarti et al. [9] proved that the communication complexity of the promise pairwise disjointness function in the shared blackboard model is $\Omega(k / t \log t)$.

THEOREM 3. [Theorem 2.5 in [9]] Let $f$ be the promise pairwise disjointness function. It holds that $C C_{f}(k, t)=\Omega(k / t \log t)$.

\subsection{Large Distance Codes}

Our proofs use the tool of error-correcting codes that was used in [4]. Let us define the notion of a code-mapping. Here, we use a similar definition to the one given by Arora and Barak [3] (Chapter 19, Definition 19.5, page 380, in [3]).

\section{Definition 3. [Code-mapping]}

Let $\Sigma$ be a finite set of symbols, called the alphabet. Fix three integers $d \geq 1, L \geq 1$ and $M \geq L$. For two strings $x, y \in \Sigma^{M}$, the distance of $x$ and $y$, denoted byd $(x, y)$, is equal to $\left|\left\{i \in[M] \mid x_{i} \neq y_{i}\right\}\right|$.

A code-mapping with parameters $(L, M, d, \Sigma)$ is a function $C$ : $\Sigma^{L} \rightarrow \Sigma^{M}$, such that for every $x \neq y \in \Sigma^{L}, d(C(x), C(y)) \geq d$.

Our proofs use the following Theorem that shows the existence of large-distance codes (Lemma 19.11 in [3]).

THEOREM 4. Let $\Sigma$ be an alphabet of size $q=|\Sigma|$. There is a code-mapping with parameters $(L, M, d, \Sigma)$, where $L \leq M \leq q$ and $d=M-L$.

One way to construct a code-mapping that proves Theorem 4 is by the so called Reed-Solomon code, which is a well-known algebraic construction for error-correcting codes. In our proofs we don't need the details of the construction, but only its existence. 


\section{MULTI-PARTY COMMUNICATION COMPLEXITY REDUCTIONS}

In this section we show how to prove lower bounds for the CONGEST model via reductions to the shared blackboard model of multi-party communication complexity. Our framework extends the framework of [8] for the 2-party case. In [8], the authors define the notion of a family of lower bound graphs for the 2-party case. In this work, we extend this notion for any arbitrary number $t \geq 2$ of players.

DEFINITION 4. [Family of Lower Bound Graphs]

Given two integers $k \geq 1, t \geq 2$, a (possibly partial) boolean function $f: \prod_{i=1}^{t}\{0,1\}^{k} \rightarrow\{$ TRUE, FALSE $\}$, and a (possibly partial) graph predicate $P$, a family of graphs

$$
\left\{G_{\bar{x}}=\left(V, E_{\bar{x}}, w_{\bar{x}}\right) \mid \bar{x}=\left(x^{1}, \cdots, x^{t}\right) \in \prod_{i=1}^{t}\{0,1\}^{k}\right\}
$$

is said to be a family of lower bound graphs with respect to $f$ and $P$ if there is a partition of the set of nodes $V=\cup_{i=1}^{t} V^{i}$ for which the following properties hold: ${ }^{2}$

(1) Only the weight of the nodes in $V^{i}$ and the existence of edges in $V^{i} \times V^{i}$ may depend on $x^{i}$

(2) $G_{\bar{x}}$ satisfies the predicate $P$ iff $f(\bar{x})=$ TRUE.

The intuition behind the definition of a family of lower bound graphs is as follows. We are given a function $f$ whose input is split among $t$ players $p^{1}, \cdots, p^{t}$, where $p^{i}$ receives a string $x^{i} \in\{0,1\}^{k}$, and a family of lower bound graphs

$$
\left\{G_{\bar{x}}=\left(V, E_{\bar{x}}, w_{\bar{x}}\right) \mid \bar{x} \in \prod_{i=1}^{t}\{0,1\}^{k}\right\}
$$

with respect to $f$ and some graph predicate $P$. In order for the players to compute the value $f\left(x^{1}, \cdots, x^{t}\right)$, they can construct the graph $G_{\bar{x}}$, where $\bar{x}=\left(x^{1}, \cdots, x^{t}\right)$, and check whether $G_{\bar{x}}$ satisfies the predicate $P$. Due to the first condition of Definition 4, each player $p^{i}$ can construct the graph induced by the nodes in $V^{i}$ without any communication with the other players. Due to the second condition of Definition $4, G_{\bar{x}}$ satisfies the predicate $P$ if and only if $f\left(x^{1}, \cdots, x^{t}\right)=$ TRUE. Hence, the problem of deciding whether $G_{\bar{x}}$ satisfies $P$ is reduced to computing the value $f\left(x^{1}, \cdots, x^{t}\right)$.

Next, we prove the following reduction theorem, which is based on a standard simulation argument. This theorem extends the reduction theorem of [8] for the 2-party case (Theorem 1 in [8]). Given a family of lower bound graphs and a graph $G_{\bar{x}}$ in it, we denote by $\operatorname{cut}\left(G_{\bar{x}}\right)$ the set of cut edges of $G_{\bar{x}}$. That is, $\operatorname{cut}\left(G_{\bar{x}}\right)=$ $E_{\bar{x}} \backslash\left(\bigcup_{i=1}^{t} V^{i} \times V^{i}\right)$.

TheOrem 5. Fix $k \geq 1, t \geq 2, f: \prod_{i=1}^{t}\{0,1\}^{k} \rightarrow\{$ TRUE, FALSE $\}$, and a graph predicate $P$. If there is a family $\left\{G_{\bar{x}}=\left(V, E_{\bar{x}}, w_{\bar{x}}\right)\right\}$ of lower bound graphs w.r.t. $f$ and $P$, then any algorithm for deciding $P$ in the CONGEST model with success probability at least $2 / 3$ requires $\Omega\left(\frac{C C_{f}(k, t)}{\left|\operatorname{cut}\left(G_{\bar{x}}\right)\right| \log |V|}\right)$ rounds.

\footnotetext{
${ }^{2}$ Throughout the paper, we use the notation $V=\dot{\cup}_{i=1}^{t} V^{i}$ to emphasize that $\left\{V^{i}\right\}_{i \in[t]}$ is a partition of $V$.
}

Proof. Let $A L G$ be a distributed algorithm in the CONGEST model that decides $P$ in $T$ rounds. We define a protocol for $f$ in the shared blackboard model, as follows. Let $\bar{x}=\left(x^{1}, \cdots, x^{t}\right) \in$ $\prod_{i=1}^{t}\{0,1\}^{k}$ be the vector of inputs of the players $p^{1}, \cdots, p^{t}$, where $p^{i}$ receives the string $x^{i}$, in the shared blackboard model. Each player $p^{i}$ constructs the part of $G_{\bar{x}}$ for the nodes in $V^{i}$. This can be done by the first condition of Definition 4 , and the fact that the $V^{i}$, s are disjoint.

The players $p^{1}, \cdots, p^{t}$ simulate $A L G$, where each player $p^{i}$ simulates the nodes in $V^{i}$, as follows. All the messages that are sent on edges in $V^{i} \times V^{i}$ are simulated by player $p^{i}$, without any communication with the other players. All the other messages, the ones that are sent on edges in $\operatorname{cut}\left(G_{\bar{x}}\right)=E_{\bar{x}} \backslash\left(\bigcup_{i=1}^{t} V^{i} \times V^{i}\right)$, are written on the shared blackboard. That is, whenever there is a message from some node in $V^{i}$ to some node in $V^{j}$ for $i \neq j \in[t]$, player $p^{i}$ writes this message on the shared blackboard, which is visible to all the other players. In particular, it is visible to $p^{j}$ who is simulating the nodes in $V^{j}$.

After simulating the $T$ rounds of $A L G$, the players know whether $G_{\bar{x}}$ satisfies the predicate $P$, and by the second condition of Definition 4 , this reveals the information about $f(\bar{x})$. Observe that the total number of bits that are written on the blackboard are $O\left(T\left|\operatorname{cut}\left(G_{\bar{x}}\right)\right| \log |V|\right)$. This is because an algorithm in the CONGEST model sends at most $O(\log |V|)$ bits on each edge in each round, and the only messages that are written on the blackboard are the ones that are sent on the edges in $\operatorname{cut}\left(G_{\bar{x}}\right)$. Hence, the communication complexity of $f$ is at most $O\left(T\left|\operatorname{cut}\left(G_{\bar{x}}\right)\right| \log |V|\right)$ and therefore, $T=\Omega\left(\frac{C C_{f}(k, t)}{\left|\operatorname{cut}\left(G_{\bar{x}}\right)\right| \log |V|}\right)$.

Our hardness results use families of lower bound graphs with respect to the promise pairwise disjointness function and a gap predicate $P$. We formalize such families in Definition 6. First, we formally define the notion of $\gamma$-approximation to maximum independent set.

\section{DEFINITION 5. [ $\gamma$-approximation to maximum independent} set]

Let $G=(V, E, w)$ be a vertex-weighted graph with weight function $w$, and let OPT be the value of an optimal solution to maximum independent set. ${ }^{3}$ An independent set $I$ in $G$ is $\gamma$-approximation to maximum independent set if $w(I) \geq O P T / \gamma$.

\section{DEFINITION 6. [ $\gamma$-approximate MaxIS family oflower bound} graphs]

Fix $0 \leq \gamma \leq 1, \beta>0$. Let $P$ be a graph predicate that distinguishes between graphs of maximum independent set of weight at least $\beta$, and graphs of maximum independent set of weight at most $\gamma \cdot \beta$. A family of graphs is called a $\gamma$-approximate MaxIS family of lower bound graphs if it is a family of lower bound graphs with respect to the promise pairwise disjointness function and the graph predicate $P$.

The following corollary follows from Theorems 3 and 5 .

\footnotetext{
${ }^{3}$ Throughout the paper, for a subset of nodes $U \subseteq V$, we denote by $w(U)=$ $\sum_{v \in U} w(v)$.
} 
COROLlary 1. Let $k \geq 1, t \geq 2$ be two integers. If there is a $\gamma$-approximate MaxIS family of graphs

$$
\left\{G_{\bar{x}}=\left(V, E_{\bar{x}}, w_{\bar{x}}\right) \mid \bar{x} \in \prod_{i=1}^{t}\{0,1\}^{k}\right\}
$$

with respect to Promise Set Disjointness function (Definition 2), then any algorithm for $\gamma$-approximation of maximum independent set in the CONGEST model with success probability at least $2 / 3$ requires $\Omega\left(k /\left(t \log t \cdot\left|\operatorname{cut}\left(G_{\bar{x}}\right)\right| \log |V|\right)\right)$ rounds.

\section{LINEAR LOWER BOUND}

In this section we prove the following theorem.

Theorem 1 For any constant $0<\epsilon<1 / 2$, any algorithm that finds $a(1 / 2+\epsilon)$-approximation to maximum independent set in the CONGEST model requires $\Omega\left(n / \log ^{3} n\right)$ rounds.

To prove Theorem 1 , we construct a $(1 / 2+\epsilon)$-approximate MaxIS family of lower bound graphs

$$
\left\{G_{\bar{x}}=\left(V, E_{\bar{x}}, w_{\bar{x}}\right) \mid \bar{x} \in \prod_{i=1}^{t}\{0,1\}^{k}\right\}
$$

\subsection{The family of lower bound graphs}

We start by describing a fixed graph construction $G=(V, E, w)$, and then we describe how to get from $G$ and a vector of strings $\bar{x} \in \prod_{i=1}^{t}\{0,1\}^{k}$ the graph $G_{\bar{x}}=\left(V, E_{\bar{x}}, x_{\bar{x}}\right)$, which gives a family of graphs $\left\{G_{\bar{x}}=\left(V, E_{\bar{x}}, w_{\bar{x}}\right) \mid \bar{x} \in \prod_{i=1}^{t}\{0,1\}^{k}\right\}$.

Our fixed graph construction $G$ contains $t$ copies of a fixed base graph $H$. We start by describing the base graph $H$.

Some notations: Let $k, \alpha, \ell$ be three positive integers that are to be chosen later such that $(\ell+\alpha)^{\alpha}=k$, and $\ell \gg \alpha$. Let $C$ be a code-mapping given by Theorem 4 with parameters $(\alpha, \ell+\alpha, \ell, \Sigma)$, where $\Sigma=\{1, \cdots, \ell+\alpha\}$. Observe that $k=|\Sigma|^{\alpha}$. Hence, we order the elements in $\Sigma^{\alpha}$ by an arbitrary ordering, and for $m \in[k]$, we denote by $C(m)$ the code-mapping of the $m$ 'th element in $\Sigma^{\alpha}$.

Description of $H=\left(V_{H}, E_{H}\right)$ : The set of nodes $V_{H}$ contains a clique of size $k$, denoted by $A=\left\{v_{1}, \ldots, v_{k}\right\}$, and $\ell+\alpha$ cliques, $C_{1}, \cdots, C_{(\ell+\alpha)}$, each of size $\ell+\alpha$. For each $h \in[\ell+\alpha]$, the nodes in $C_{h}$ are denoted by $C_{h}=\left\{\sigma_{(h, 1)}, \cdots, \sigma_{(h, \ell+\alpha)}\right\}$. We call the cliques $C_{1}, \cdots, C_{\ell+\alpha}$ the code gadget, and we denote this set of nodes by

$$
\text { Code }=\bigcup_{h=1}^{\ell+\alpha} C_{h}
$$

The reason that these cliques are called the code-gadget is as follows. Given a code-word $w \in \Sigma^{\ell+\alpha}$, we can represent $w$ by $\ell+\alpha$ nodes $u_{1} \in C_{1}, u_{2} \in C_{2}, \cdots, u_{\ell+\alpha} \in C_{\ell+\alpha}$, where $u_{h} \in C_{h}$ corresponds to the $h$ 'th position in $w$. That is, $u_{h}=\sigma_{\left(h, w_{h}\right)}$, where $w_{h}$ is the value in the $h$ 'th position in $w$. For any $m \in[k]$, we denote by Code $m$ the set of nodes that corresponds to the code-word $C(m) \in \Sigma^{\ell+\alpha}$, and we connect $v_{m} \in A$ to all the nodes in Code $\backslash$ Code $e_{m}$.

This concludes the description of $H$ (we provide an illustration in Figure 1). More formally, the graph $H=\left(V_{H}, E_{H}\right)$ is defined

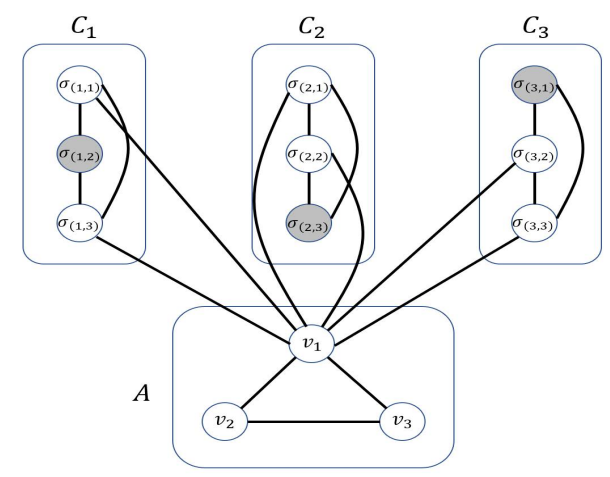

Figure 1: An example of the base graph $H$, where $\ell=2, \alpha=1$. $A$ is a clique of $k=(\ell+\alpha)^{\alpha}=3$ nodes, and there are $\ell+\alpha=$ 3 cliques $C_{1}, C_{2}$, and $C_{3}$, each of size $\ell+\alpha$. In this example, we assume that the code-mapping of $1, C(1)=$ " $2,3,1$ ", and therefore, $v_{1}$ in connected to all the nodes in Code $=C_{1} \cup C_{2} \cup$ $C_{3}$, except for the nodes in $\operatorname{Code}_{1}=\left\{\sigma_{(1,2)}, \sigma_{(2,3)}, \sigma_{(3,1)}\right\}$. The other edges between $\left\{v_{2}, v_{3}\right\} \times$ Code are omitted in this figure, for clarity.

as follows. Given a clique $C$, we denote by $E(C)$ the set of all the possible edges between nodes in $C$.

$$
\begin{aligned}
& V_{H}=A \cup \text { Code } \\
& E_{H}=E(A) \cup\left\{\left\{v_{m}, u\right\} \mid v_{m} \in A, u \in \operatorname{Code} \backslash \operatorname{Code}_{m}\right\} \bigcup_{h=1}^{\ell+\alpha} E\left(C_{h}\right)
\end{aligned}
$$

Obtaining the fixed graph construction $G$ from $H$ : Now we are ready to describe the fixed graph construction $G=(V, E)$. Let $t \geq 2$. There are $t$ copies of $H$ in $G$, denoted by $H^{1}, \cdots, H^{t}$. In order to distinguish between nodes in different $H^{i}$ 's, we add a superscript $i$ for the nodes in $H^{i}$. That is, for each $i \in[t]$, $H^{i}=\left(V^{i}, E^{i}\right)$ contains a clique and a code-gadget, where the clique is denoted by $A^{i}=\left\{v_{1}^{i}, \cdots, v_{k}^{i}\right\}$, the code-gadget is denoted by $C^{i} e^{i}$, the cliques in the code-gadget are denoted by $C_{1}^{i}, \cdots, C_{\ell+\alpha}^{i}$, and for any $h \in[\ell+\alpha]$, the nodes in $C_{h}^{i}$ are denoted by $C_{h}^{i}=\left\{\sigma_{(h, 1)}^{i}, \cdots, \sigma_{(h, \ell+\alpha)}^{i}\right\}$. Similarly, $\operatorname{Cod} e_{m}^{i}$ denotes the set of nodes in $\bigcup_{i=1}^{\ell+\alpha} C_{h}^{i}$ that corresponds to the code-word $C_{m}$. That is, let $w=C(m)$, we have that $\operatorname{Cod} e_{m}^{i}=\left\{\sigma_{\left(1, w_{1}\right)}^{i}, \cdots, \sigma_{\left(\ell+\alpha, w_{\ell+\alpha}\right)}^{i}\right\}$.

It remains to describe the connections between $H^{i}$ and $H^{j}$, for any $i \neq j \in[t]$. For any $h \in[\ell+\alpha]$, we add all the possible edges between $C_{h}^{i}$ and $C_{h}^{j}$ except for the natural perfect matching between $C_{h}^{i}$ and $C_{h}^{j}$, i.e., $\left\{\left\{\sigma_{(h, r)}^{i}, \sigma_{(h, r)}^{j}\right\} \mid r \in[\ell+\alpha]\right\}$. More formally, we add the following edges for any $i \neq j \in[t]$ and any $h \in[\ell+\alpha]$,

$$
\left\{\{u, v\} \mid u \in C_{h}^{i}, v \in C_{h}^{j}\right\} \backslash\left\{\left\{\sigma_{(h, r)}^{i}, \sigma_{(h, r)}^{j}\right\} \mid r \in[\ell+\alpha]\right\}
$$

We provide an illustration for these connections in the full version of the paper [12], Figure 2. This concludes our fixed graph construction $G$, and we proceed to describing $G_{\bar{x}}$. An illustration for the full 
construction is provided in Figure 3 in the full version of the paper [12].

Obtaining $G_{\bar{x}}$ from $G$ and $\bar{x}$ :

Given $\bar{x}=\left(x^{1}, \cdots, x^{t}\right) \in \prod_{i=1}^{t}\{0,1\}^{k}$.

The graph $G_{\bar{x}}=\left(V, E, w_{\bar{x}}\right)$ is defined as follows. The sets of nodes and edges of $G_{\bar{x}}$ are exactly as in $G$. The weights of nodes in $G_{\bar{x}}$ are defined as follows. Let $i \in[t], m \in[k]$, and $v_{m}^{i} \in A^{i}$,

$$
w\left(v_{m}^{i}\right)= \begin{cases}\ell & \text { if } x_{m}^{i}=1 \\ 1 & \text { otherwise }\end{cases}
$$

All the other nodes in $G_{\bar{x}}$ are of weight 1 . That is, for any $u \in$ $V \backslash \bigcup_{i=1}^{t} A^{i}, w(u)=1$.

This concludes the description of $G_{\bar{x}}$. Before we proceed to proving that $G_{\bar{x}}$ is a family of lower bound graphs, we provide three useful properties of $G_{\bar{x}}$ that are used in the proof.

Property 1. For any $m \in[k]$, it holds that $\left(\cup_{i=1}^{t} \operatorname{Code} e_{m}^{i}\right) \cup$ $\left\{v_{m}^{i} \mid i \in[t]\right\}$ is an independent set.

Proof. First, observe that the nodes in $\left\{v_{m}^{i} \mid i \in[t]\right\}$ are independent. This is because $v_{m}^{i} \in A^{i}$, and there are no edges between $A^{i}$ and $A^{j}$ for any $i \neq j$. There are also no edges between $A^{i}$ and $\operatorname{Code} e^{j}$, for any $i \neq j$. Furthermore, for any $i \in[t]$ and any $m \in[k]$, it holds that $\left\{v_{m}^{i}\right\} \cup \operatorname{Cod} e_{m}^{i}$ is an independent set. This is because $v_{m}^{i}$ is connected only to the nodes in $\operatorname{Code}^{i} \backslash \operatorname{Code}_{m}^{i}$. Finally, let $w=C(m)$ be the code-mapping of $m$. Since for any $i \neq j$, we have that $\operatorname{Cod} e_{m}^{i}=\left\{\sigma_{\left(1, w_{1}\right)}^{i}, \cdots \sigma_{\left(\ell+\alpha, w_{\ell+\alpha}\right)}^{i}\right\}$, and $\operatorname{Code} e_{m}^{j}=\left\{\sigma_{\left(1, w_{1}\right)}^{j}, \cdots \sigma_{\left(\ell+\alpha, w_{\ell+\alpha}\right)}^{j}\right\}$, and $\sigma_{(h, r)}^{i}$ is not connected to $\sigma_{(h, r)}^{j}$ for any $h, r \in[\ell+\alpha]$, we have that $\bigcup_{i=1}^{t} \operatorname{Code}_{m}^{i}$ is an independent set. Hence, the union $\left(\bigcup_{i=1}^{t} \operatorname{Code} e_{m}^{i}\right) \cup\left\{v_{m}^{i} \mid i \in[t]\right\}$ is an independent set.

Property 2. For any $i \neq j \in[t]$, and any $m_{1} \neq m_{2} \in[k]$, the bipartite graph (Code ${ }_{m_{1}}^{i}, \operatorname{Code}_{m_{2}}^{j}$ ) contains a matching of size at least $\ell$.

Proof. Let $w^{1}=C\left(m_{1}\right)$ be the code-mapping of $m_{1}$, and let $w^{2}=C\left(m_{2}\right)$ be the code-mapping of $m_{2}$. Given $h, r \in[\ell+\alpha]$, observe that $\sigma_{(h, r)}^{i}$ is connected to all the nodes in $C_{h}^{j} \backslash\left\{\sigma_{(h, r)}^{j}\right\}$. Hence, since the distance between $w^{1}$ and $w^{2}$ is at least $\ell$, there are at least $\ell$ positions $h \in[\ell+\alpha]$ for which $w_{h}^{1} \neq w_{h}^{2}$, and therefore, there are at least $\ell$ positions $h \in[\ell+\alpha]$ for which it holds that $\sigma_{\left(h, w_{h}^{1}\right)}^{i}$ is connected to $\sigma_{\left(h, w_{h}^{2}\right)}^{j}$, where $w_{h}^{1}$ is the $h^{\prime}$ th position in $w^{1}$ and $w_{h}^{2}$ is the $h^{\prime}$ th position in $w^{2}$.

Property 3. Let $i \neq j \in[t]$, let $m_{1} \neq m_{2} \in[k]$, and let $I$ be any independent set. Let $w^{1}=C\left(m_{1}\right)$ be the code mapping of $m_{1}$, and let $w^{2}=C\left(m_{2}\right)$ be the code-mapping of $m_{2}$. The number of positions $h \in[\ell+\alpha]$ for which it holds that $\sigma_{\left(h, w_{h}^{1}\right)}^{i} \in I$ and $\sigma_{\left(h, w_{h}^{2}\right)}^{j} \in I$ is at most $\alpha$.

Proof. By Property 2, the bipartite graph ( $\operatorname{Code} e_{m_{1}}^{i}, \operatorname{Code}_{m_{2}}^{j}$ ) contains a matching of size at least $\ell$. Therefore, there are at least $\ell$ positions $h \in[\ell+\alpha]$ for which $I$ contains at most one of the nodes $\sigma_{\left(h, w_{h}^{1}\right)}^{i}$ and $\sigma_{\left(h, w_{h}^{2}\right)}^{j}$. This leaves at most $\alpha$ other positions for which $I$ can contain both $\sigma_{\left(h, w_{h}^{1}\right)}^{i}$ and $\sigma_{\left(h, w_{h}^{2}\right)}^{j}$.

\section{2 $G_{\bar{x}}$ is a $(1 / 2+\epsilon)$-approximate MaxIS family of lower bound graphs}

In this section we show that there is a constant $t>2$ for which $G_{\bar{x}}$ is a $(1 / 2+\epsilon)$-approximate MaxIS family of graphs. We start with a slightly weaker statement for $t=2$, which is later used in the proof for $t>2$.

4.2.1 Warm-up: $t=2$. In this section we prove the following lemma.

LEMMA 1. For $t=2$, and for any constant $\epsilon>0$, it holds that $\left\{G_{\bar{x}}=\left(V, E_{\bar{x}}, w_{\bar{x}}\right) \mid \bar{x} \in \prod_{i=1}^{t}\{0,1\}^{k}\right\}$ is a $(3 / 4+\epsilon)$-approximate MaxIS family of lower bound graphs.

For the rest of this subsection, we assume that $t=2$. Lemma 1 is a corollary of Claims 1 and 2 .

Claim 1. For any

$g_{\left(x^{1}, x^{2}\right)} \in\left\{G_{\left(x^{1}, x^{2}\right)}=\left(V, E_{\left(x^{1}, x^{2}\right)}, w_{\left(x^{1}, x^{2}\right)}\right) \mid\left(x^{1}, x^{2}\right) \in \prod_{i=1}^{2}\{0,1\}^{k}\right\}$ , if $x^{1}$ and $x^{2}$ are not disjoint, then $g_{\left(x^{1}, x^{2}\right)}$ contains an independent set of weight at least $4 \ell+2 \alpha$.

Proof. Since the sets are not disjoint, there is an $m \in[k]$ for which $x_{m}^{1}=x_{m}^{2}=1$. Therefore, the weight of each of the nodes $v_{m}^{1}$ and $v_{m}^{2}$ is $\ell$. By Property 1 , the set $\left\{v_{m}^{1}\right\} \cup\left\{v_{m}^{2}\right\} \cup \operatorname{Cod} e_{m}^{1} \cup \operatorname{Cod} e_{m}^{2}$ is independent, and observe that its weight is $4 \ell+2 \alpha$.

Claim 2. For any

$g_{\left(x^{1}, x^{2}\right)} \in\left\{G_{\left(x^{1}, x^{2}\right)}=\left(V, E_{\left(x^{1}, x^{2}\right)}, w_{\left(x^{1}, x^{2}\right)}\right) \mid\left(x^{1}, x^{2}\right) \in \prod_{i=1}^{2}\{0,1\}^{k}\right\}$

, if $x^{1}$ and $x^{2}$ are disjoint, then any independent set $I$ in $g_{\left(x^{1}, x^{2}\right)}$ is of weight at most $3 \ell+2 \alpha+1$.

Proof. The proof is by the following simple case analysis.

(1) $I$ contains at most one node of weight $\ell$ : In this case, the node of weight $\ell$ must be either in the clique $A^{1}$ or in the clique $A^{2}$. Assume without loss of generality that this node is in $A^{1}$. Observe that we can take at most one node of weight 1 from $A^{2}$. Furthermore, since each of $\operatorname{Cod} e^{1}$ and $\operatorname{Code}^{2}$ is a union of $\ell+\alpha$ cliques, we cannot construct an independent set in $\operatorname{Code}^{1} \cup \operatorname{Code}^{2}$ of weight larger than $2(\ell+\alpha)$, it follows that the weight of $I$ cannot be larger than $3 \ell+2 \alpha+1$.

(2) $I$ contains two nodes of weight $\ell$ : This implies that $I$ contains one node $v_{m_{1}}^{1} \in A^{1}$ of weight $\ell$ and another node $v_{m_{2}}^{2} \in A^{2}$ of weight $\ell$, where $m_{1}, m_{2} \in[k]$. Since the strings $x^{1}, x^{2}$ are disjoint, it must be the case that $m_{1} \neq m_{2}$. Furthermore, since $v_{m_{1}}^{1}$ is connected to the nodes in $\operatorname{Code} e^{1} \backslash \operatorname{Code}_{m_{1}}^{1}$, and $v_{m_{2}}^{2}$ is connected to the nodes in $\operatorname{Code} e^{2} \backslash \operatorname{Code}_{m_{2}}^{2}$, it remains to show that $\left|I \cap\left(\operatorname{Code}_{m_{1}}^{1} \cup \operatorname{Code} e_{m_{2}}^{2}\right)\right| \leq \ell+2 \alpha$. By Property 2, $\left(\operatorname{Code}_{m_{1}}^{1}, \operatorname{Code}_{m_{2}}^{2}\right)$ contains a matching of size at least $\ell$, and since $\left|\operatorname{Code}_{m_{1}}^{1} \cup \operatorname{Code}_{m_{2}}^{2}\right|=(2 \ell+2 \alpha)$, this implies that $\mid I \cap$ 
$\left(\operatorname{Code}_{m_{1}}^{1} \cup \operatorname{Code}_{m_{2}}^{2}\right) \mid \leq \ell+2 \alpha$. To conclude, in this case, $I$ contains 2 nodes of weight $\ell$ and at most $\ell+2 \alpha$ nodes of weight 1 . In total, the weight of $I$ is at most $3 \ell+2 \alpha$.

Notice that $I$ cannot contain more than 2 elements of weight $\ell$ since the elements of weight $\ell$ form two disjoint cliques.

Proof of Lemma 1. Claims 1 and 2 imply that

$$
\left\{G_{\bar{x}}=\left(V, E_{\bar{x}}, w_{\bar{x}}\right) \mid \bar{x} \in \prod_{i=1}^{2}\{0,1\}^{k}\right\}
$$

is a family of lower bound graphs with respect to the set disjointness function and the graph predicate that distinguishes between graphs of maximum independent set at least $4 \ell+2 \alpha$ and graphs of maximum independent set at most $3 \ell+2 \alpha+1$.

We set $\ell=\log k-\log k / \log \log k, \alpha=\log k / \log \log k$. Hence $(\ell+\alpha)^{\alpha}=k$ as desired. Since the dominating terms in the two cases are $4 \ell$ and $3 \ell$, it follows that for any constant $\epsilon>0$,

$$
\left\{G_{\bar{x}}=\left(V, E_{\bar{x}}, w_{\bar{x}}\right) \mid \bar{x} \in \prod_{i=1}^{2}\{0,1\}^{k}\right\}
$$

is a $(3 / 4+\epsilon)$-approximate MaxIS family of graphs. ${ }^{4}$

4.2.2 Hardness Amplification using $t>2$ Players. In this section we prove the following lemma, whose proof is a corollary of Claims 3 and 5, where in between Claims 3 and 5 we have a helper claim (Claim 4) and a corollary of it.

Lемма 2. For any constant $\epsilon>0$, there is a constant $t>2$ for which it holds that $\left\{G_{\bar{x}}=\left(V, E_{\bar{x}}, w_{\bar{x}}\right) \mid \bar{x} \in \prod_{i=1}^{t}\{0,1\}^{k}\right\}$ is a $(1 / 2+\epsilon)$-approximate MaxIS family of lower bound graphs.

CLaim 3. For any positive integer t, and any

$$
g_{\bar{x}} \in\left\{G_{\bar{x}}=\left(V, E_{\bar{x}}, w_{\bar{x}}\right) \mid \bar{x} \in \prod_{i=1}^{t}\{0,1\}^{k}\right\}
$$

, if there is an $m \in[k]$ for which it holds that $x_{m}^{1}=\cdots=x_{m}^{t}=1$, then $g_{\bar{x}}$ contains an independent set of weight at least $t(2 \ell+\alpha)$.

Proof. Observe that for any $i \in[t]$, it holds that $w\left(v_{m}^{i}\right)=\ell$. Furthermore, by Property $1,\left(\cup_{i=1}^{t} \operatorname{Cod} e_{m}^{i}\right) \cup\left\{v_{m}^{i} \mid i \in[t]\right\}$ is an independent set, and it is of weight $2 t \ell+t \alpha$.

Claim 4. For any positive integer $t$, let $m_{1}, m_{2}, \cdots, m_{t}$ be any $t$ pairwise distinct values in $[k]$. For any independent set $I$, if $\left\{v_{m_{i}}^{i} \mid\right.$ $i \in[t]\} \subseteq I$, then

$$
\left|I \cap\left(\bigcup_{i=1}^{t} \operatorname{Cod} e_{m_{i}}^{i}\right)\right| \leq \ell+\alpha t^{2}
$$

Proof. Let us start with some notations. Let $w^{i}=C\left(m_{i}\right)$ be the code-mapping of $m_{i}$. Hence, we have that

$$
\operatorname{Code}_{m_{i}}^{i}=\left\{\sigma_{\left(1, w_{1}^{i}\right)}^{i}, \cdots, \sigma_{\left(\ell+\alpha, w_{\ell+\alpha}^{i}\right)}^{i}\right\}
$$

. Furthermore, let $S=\left\{h \in[\ell+\alpha]\left|\sum_{i=1}^{t}\right| I \cap \sigma_{\left(h, w_{h}^{i}\right)}^{i} \mid \leq 1\right\}$, $\bar{S}=[\ell+\alpha] \backslash S$. That is, $S$ is the set of values $h \in[\ell+\alpha]$ for which

${ }^{4}$ In fact, by slightly changing the parameters $\ell$ and $\alpha$, the claim holds for any $\epsilon=$ $\Omega(1 / \log k)$. the independent set $I$ contains at most one node in $\bigcup_{i=1}^{t}\left\{\sigma_{\left(h, w_{h}^{i}\right)}^{i}\right\}$. Finally, let $\psi_{i, j}^{h}$ be an indicator defined as follows.

$$
\psi_{i, j}^{h}= \begin{cases}1 & \text { if } \sigma_{\left(h, w_{h}^{i}\right)}^{i} \in I \text { and } \sigma_{\left(h, w_{h}^{j}\right)}^{j} \in I \\ 0 & \text { otherwise }\end{cases}
$$

By Property 3, for any $i \neq j \in[t]$, it holds that $\sum_{h \in[\alpha+\ell]} \psi_{i, j}^{h} \leq \alpha$. Hence,

$$
\begin{aligned}
& \left|I \cap\left(\bigcup_{i=1}^{t} \operatorname{Cod} e_{m_{i}}^{i}\right)\right|=\sum_{i=1}^{t}\left|I \cap \operatorname{Cod}_{m_{i}}^{i}\right|=\sum_{i=1}^{t}\left|I \cap\left(\bigcup_{h=1}^{\ell+\alpha}\left\{\sigma_{\left(h, w_{h}^{i}\right)}^{i}\right\}\right)\right| \\
& =\sum_{i=1}^{t} \sum_{h=1}^{\ell+\alpha}\left|I \cap\left\{\sigma_{\left(h, w_{h}^{i}\right)}^{i}\right\}\right|=\sum_{h=1}^{\ell+\alpha} \sum_{i=1}^{t}\left|I \cap\left\{\sigma_{\left(h, w_{h}^{i}\right)}^{i}\right\}\right| \\
& =\left(\sum_{h \in S} \sum_{i=1}^{t}\left|I \cap\left\{\sigma_{\left(h, w_{h}^{i}\right)}^{i}\right\}\right|\right)+\left(\sum_{h \in \bar{S}} \sum_{i=1}^{t}\left|I \cap\left\{\sigma_{\left(h, w_{h}^{i}\right)}^{i}\right\}\right|\right) \\
& \leq\left(\sum_{h \in S} \sum_{i=1}^{t}\left|I \cap\left\{\sigma_{\left(h, w_{h}^{i}\right)}^{i}\right\}\right|\right)+\left(\sum_{h \in \bar{S}} \sum_{i \neq j \in[t]} 2 \psi_{i, j}^{h}\right) \\
& =\left(\sum_{h \in S} \sum_{i=1}^{t}\left|I \cap\left\{\sigma_{\left(h, w_{h}^{i}\right)}^{i}\right\}\right|\right)+\left(\sum_{i \neq j \in[t]} \sum_{h \in \bar{S}} 2 \psi_{i, j}^{h}\right) \\
& \leq \ell+\alpha+2 \alpha \cdot t(t-1) / 2 \leq \ell+\alpha t^{2}
\end{aligned}
$$

Where (1), (2), and (3) are straightforward. (4) holds because for any $h \in \bar{S}$, there are at least two indices $i \neq j \in[t]$, for which it holds that $\sigma_{\left(h, w_{h}^{i}\right)}^{i} \in I$ and $\sigma_{\left(h, w_{h}^{j}\right)}^{j} \in I$. And in particular if there are, say, $t$ such indexes, the amount of pairs of indexes $i, j$ for which $\psi_{i, j}^{j}=1$ is atleast $\frac{t(t-1)}{2} \geq \frac{t}{2}$. (5) holds by changing the summation order of the second sum. (6) holds because for any $h \in S$, $\sum_{i=1}^{t}\left|I \cap\left\{\sigma_{\left(h, w_{h}^{i}\right)}^{i}\right\}\right| \leq 1$, and by Property $3, \sum_{h \in[\ell+\alpha]} \psi_{i, j}^{h} \leq \alpha$.

Corollary 2. For any positive integer $t$, let $m_{1}, m_{2}, \cdots, m_{t}$ be any $t$ distinct values in $[k]$. For any independent set $I$, if $\left\{v_{m_{i}}^{i} \mid i \in\right.$ $[t]\} \subseteq I$, then

$$
w(I) \leq(t+1) \ell+\alpha t^{2}
$$

Proof. Since each $v_{m_{i}}^{i}$ is connected to all the nodes in $\operatorname{Code} e^{i} \backslash$ $\operatorname{Code}_{m_{i}}^{i}$, we have that

$$
\begin{aligned}
& w(I)=w\left(I \cap\left(\bigcup_{i=1}^{t} A_{i}\right)\right)+w\left(I \cap\left(\bigcup_{i=1}^{t} \operatorname{Cod} e^{i}\right)\right) \\
& =\left(\sum_{i=1}^{t} w\left(v_{m_{i}}^{i}\right)\right)+w\left(I \cap\left(\bigcup_{i=1}^{t} \operatorname{Cod}_{m_{i}}^{i}\right)\right) \\
& \leq t \ell+\ell+\alpha t^{2}=(t+1) \ell+\alpha t^{2}
\end{aligned}
$$

Claim 5. For any positive integer t, and any

$$
g_{\bar{x}} \in\left\{G_{\bar{x}}=\left(V, E_{\bar{x}}, w_{\bar{x}}\right) \mid \bar{x} \in \prod_{i=1}^{t}\{0,1\}^{k}\right\}
$$


, if the strings $x^{1}, \cdots, x^{t}$ are pairwise disjoint, then the weight of any independent set is at most $(t+1) \ell+\alpha t^{2}$.

Proof. The proof is by induction on $t$, where the base case of $t=1$ is straightforward (even the case of $t=2$ was already proved in Claim 2). We assume correctness for $t-1$, and prove correctness for $t$. Let $I$ be an independent set in $g_{\bar{x}}$. Recall that $A^{i}$ is a clique and therefore $\left|I \cap A^{i}\right| \leq 1$. The proof is by the following case analysis.

(1) There is some $i \in[t]$ for which it holds that $I \cap A^{i}$ is either empty, or contains a node of weight 1 : Observe that in this case, $w\left(I \cap V^{i}\right) \leq \ell+\alpha+1$. This is because any independent set contains at most $\ell+\alpha$ nodes in $\operatorname{Cod} e^{i}=V^{i} \backslash A^{i}$. Furthermore, by the inductive hypothesis on the graph induced by the nodes in $\bigcup_{j \in[t] \backslash\{i\}} V^{j}$, we have that $w(I) \leq t \ell+\alpha(t-1)^{2}+$ $\ell+\alpha+1 \leq(t+1) \ell+\alpha\left(t^{2}-2 t+1\right)+\alpha+1<(t+1) \ell+\alpha\left(t^{2}\right)$, where the last inequality holds since $\alpha \geq 1$, and $t>2$.

(2) For any $i \in[t], I \cap A^{i}$ contains a node of weight $\ell$, denoted by $v_{m_{i}}^{i}$ : This case is proved directly, without applying the inductive hypothesis, as follows. First, since the strings $x^{1}, \cdots, x^{t}$ are pairwise disjoint, it must be the case that for any $i \neq j \in[t], m_{i} \neq m_{j}$. This is because $w\left(v_{m_{i}}^{i}\right)=\ell$ if and only if $x_{m_{i}}^{i}=1$, and if $m_{i}=m_{j}$, it would imply that $x^{i}$ and $x^{j}$ are not disjoint. Hence, by Corollary 2, we have that

$$
w(I) \leq(t+1) \ell+\alpha t^{2}
$$

As desired.

Proof of Lemma 2. Claims 3 and 5 imply that

$$
\left\{G_{\bar{x}}=\left(V, E_{\bar{x}}, w_{\bar{x}}\right) \mid \bar{x} \in \prod_{i=1}^{t}\{0,1\}^{k}\right\}
$$

is a family of lower bound graphs with respect to the pairwise disjointness function and the graph predicate that distinguishes between graphs of maximum independent set at least $t(2 \ell+\alpha)$ and graphs of maximum independent set at most $(t+1) \ell+\alpha \cdot t^{2}$.

Recall that $\ell=\log k-\log k / \log \log k, \alpha=\log k / \log \log k$. Which implies that the graph predicate distinguishes between independent sets of weight at least $t(2 \log k-2 \log k / \log \log k+\log k / \log \log k)=$ $2 t \log k-\log k / \log \log k \geq(2 t-1) \log k$ and independent sets of weight at most $(t+1)(\log k-\log k / \log \log k)+t^{2}(\log k / \log \log k) \leq$ $(t+2) \log k$, for any constant $t$ and $k \gg t$. Hence, for any constant $\epsilon>0$, we choose $t=3 / \epsilon$ (or the first integer larger than $3 / \epsilon$, if it is not an integer). This implies that for any constant $\epsilon>0$, there is a constant $t$ for which $\left\{G_{\bar{x}}=\left(V, E_{\bar{x}}, w_{\bar{x}}\right) \mid \bar{x} \in \prod_{i=1}^{t}\{0,1\}^{k}\right\}$ is a $(1 / 2+\epsilon)$-approximate MaxIS family of graphs.

Proof of Theorem 1. Observe that $k=\Theta(n)$, where $n=|V|$. Furthermore, $\left\{G_{\bar{x}}=\left(V, E_{\bar{x}}, w_{\bar{x}}\right) \mid \bar{x} \in \prod_{i=1}^{t}\{0,1\}^{k}\right\}$ is a $(1 / 2+\epsilon)$ approximate MaxIS family of graphs, where the partition of the set of nodes that is needed for Definition 4 is $V=\bigcup_{i=1}^{t} V^{i}$. Hence, by Corollary 1 and the fact that $\left|\operatorname{cut}\left(G_{\bar{x}}\right)\right|=t^{2} \log ^{2} k=\Theta\left(\log ^{2} k\right)$, any algorithm for finding a $(1 / 2+\epsilon)$-approximation to maximum independent set in the CONGEST model with success probability at least $2 / 3$ requires $\Omega\left(k /\left(t \log t \cdot\left|\operatorname{cut}\left(G_{\bar{x}}\right)\right| \log |V|\right)\right)=\Omega(n /(t \log t$. $\left.\log ^{3} n\right)=\Omega\left(n / \log ^{3} n\right)$ rounds.
REMARK 1. While our hard instances in the proof of Theorem 1 are weighted, it is easy to extend the argument for unweighted graphs as well, by losing a logarithmic factor in the lower bound (in terms of the number of rounds), as follows. For every node $v$ of weight $\ell$, we replace $v$ by an independent set of size $\ell$, denoted by $I(v)$. For every node $u$ that is adjacent to $v$ in our construction, if $u$ is of weight 1 , we connect all the nodes in $I(v)$ to $u$. Otherwise, if $u$ is of weight $\ell$, it means that it is replaced by an independent set of size $\ell$, denoted by $I(u)$. We connect $I(v)$ to $I(u)$ by a bi-clique (a full bipartite graph). The proof that the converted construction yields a hardness of $(1 / 2+\epsilon)$ approximation follows from a similar case analysis to the one provided for the weighted case. Since the number of nodes in the unweighted construction in $n=\Theta(k \ell)=\Theta(k \log k)$ rather than $\Theta(k)$, in terms of the number of rounds, we lose a logarithmic factor in the lower bound compared to the weighted case.

\section{QUADRATIC LOWER BOUND}

In this section we prove the following theorem.

Theorem 2 For any constant $0<\epsilon<1 / 4$, any algorithm that finds a $(3 / 4+\epsilon)$-approximation to maximum independent set in the CONGEST model requires $\Omega\left(n^{2} / \log ^{3} n\right)$ rounds.

To prove Theorem 2, we construct a $(3 / 4+\epsilon)$-approximate MaxIS family of lower bound graphs

$$
\left\{F_{\bar{x}}=\left(V, E_{\bar{x}}, w_{\bar{x}}\right) \mid \bar{x} \in \prod_{i=1}^{t}\{0,1\}^{k^{2}}\right\}
$$

. Observe that unlike the previous section, the length of the strings in $\bar{x}$ is $k^{2}$ rather than $k$. In our graph construction, similarly to the previous section, $k=\Theta(n)$. Hence, having the length of the strings being $k^{2}$ allows us to achieve a near-quadratic lower bound. Our hard instances are weighted graphs, and we can extend our argument to unweighted graphs as well by losing a logarithmic factor in the lower bound (in terms of the number of rounds) in the same way as explained in Remark 1.

\subsection{The family of lower bound graphs}

We begin with describing the graph construction, $F=\left(V_{F}, E_{F}, w_{F}\right)$, and then we describe how to get from $F$ and a vector of strings $\bar{x} \in \prod_{i=1}^{t}\{0,1\}^{k^{2}}$ the graph $F_{\bar{x}}=\left(V, E_{\bar{x}}, x_{\bar{x}}\right)$. Let $G$ be the fixed graph construction defined in Section 4.1. The fixed graph construction $F$ consists of exactly two copies of $G$, denoted by $G^{1}$ and $G^{2}$. Recall that $G=\left(V_{G}, E_{G}\right)$ where $V_{G}=\bigcup_{i=1}^{t} A^{i} \cup \operatorname{Code}^{i}$. In order to distinguish between the sets of nodes that belong to $G^{1}$ and the sets of nodes that belong to $G^{2}$, we add an ordered pair as a superscript $(i, b)$, where $b \in\{1,2\}$ indicates whether the set is in $G^{1}$ or in $G^{2}$. That is, the set of nodes of $G^{1}$ is $V_{G^{1}}=\bigcup_{i=1}^{t} A^{(i, 1)} \cup \operatorname{Code}(i, 1)$, and the set of nodes of $G^{2}$ is $V_{G^{2}}=\bigcup_{i=1}^{t} A^{(i, 2)} \cup \operatorname{Code} e^{(i, 2)}$. Hence the set of nodes of $F$ is $V_{F}=\bigcup_{i=1}^{t} V^{i}$, where for any $i \in[t]$, we denote by 


$$
\begin{aligned}
& V^{i}=V^{(i, 2)} \cup V^{(i, 2)} \\
& V^{(i, 1)}=A^{(i, 1)} \cup \operatorname{Code}^{(i, 1)} \\
& V^{(i, 2)}=A^{(i, 2)} \cup \operatorname{Code}^{(i, 2)} \\
& A^{(i, 1)}=\left\{v_{m}^{(i, 1)} \mid m \in[k]\right\} \\
& A^{(i, 2)}=\left\{v_{m}^{(i, 2)} \mid m \in[k]\right\} \\
& \operatorname{Code}^{(i, 1)}=\bigcup_{h=1}^{\ell+\alpha} C_{h}^{(i, 1)} \\
& \operatorname{Code}^{(i, 2)}=\bigcup_{h=1}^{\ell+\alpha} C_{h}^{(i, 2)} \\
& C_{h}^{(i, 1)}=\left\{\sigma_{(h, 1)}^{(i, 1)}, \cdots, \sigma_{(h, \ell+\alpha)}^{(i, 1)}\right\} \\
& C_{h}^{(i, 2)}=\left\{\sigma_{(h, 1)}^{(i, 2)}, \cdots, \sigma_{(h, \ell+\alpha)}^{(i, 2)}\right\} \\
& \operatorname{Code}_{w}^{(i, 1)}=\left\{\sigma_{\left(h, w_{h}\right)}^{(i, 1)} \mid h \in[\ell+\alpha]\right\} \\
& \operatorname{Code}_{w}^{(i, 2)}=\left\{\sigma_{\left(h, w_{h}\right)}^{(i, 2)} \mid h \in[\ell+\alpha]\right\}
\end{aligned}
$$

The weight function $w_{F}$ is defined as follows. For any $v \in V_{F}$,

$$
w_{F}(v)= \begin{cases}\ell & \text { if } v \in \cup_{i=1}^{t} A^{(i, 1)} \cup A^{(i, 2)} \\ 1 & \text { otherwise }\end{cases}
$$

That is, the weight of any node in the cliques $\bigcup_{i=1}^{t} A^{(i, 1)} \cup A^{(i, 2)}$ is $\ell$, and the weight of any node in the code-gadgets $\bigcup_{i=1}^{t} \operatorname{Code} e^{(i, 1)} \cup$ Code $e^{(i, 2)}$ is 1 . Observe that unlike the previous section, the weights of the nodes don't depend on the strings in $\bar{x}$. We provide illustrations in Figures 4, 5, and 6 for the full construction in the full version of the paper [12].

Obtaining $F_{\bar{x}}$ from $F=\left(V_{F}, E_{F}, w_{F}\right)$ and $\bar{x}$ : Let $\bar{x}=\left(x^{1}, \cdots, x^{t}\right) \in$ $\prod_{i=1}^{t}\{0,1\}^{k^{2}}$. For any $x^{i}$, we index the $k^{2}$ positions in $x^{i}$ by $x_{\left(m_{1}, m_{2}\right)}^{i}$, for $m_{1}, m_{2} \in[k]$. The graph $F_{\bar{x}}$ is defined as follows. The set of nodes and the weight function remain exactly as in $F$. The set of edges contains all the edges in $F$, and the following edges in $A^{(i, 1)} \times A^{(i, 2)}$, for any $i \in[t]$.

$$
\left\{v_{m_{1}}^{(i, 1)}, v_{m_{2}}^{(i, 2)} \mid x_{\left(m_{1}, m_{2}\right)}^{i}=0\right\}
$$

That is, for any $i \in[t]$ and any $m_{1}, m_{2} \in[k]$, we add an edge between $v_{m_{1}}^{(i, 1)} \in A^{(i, 1)}$ and $v_{m_{2}}^{(i, 2)} \in A^{(i, 2)}$ if and only if $x_{\left(m_{1}, m_{2}\right)}^{i}=0$.

\section{2 $F_{\bar{x}}$ is a $(3 / 4+\epsilon)$-approximate MaxIS family of lower bound graphs}

In this section we prove the following lemma.

LемMA 3. For any constant $\epsilon>0$, there is a constant $t>2$ for which it holds that $\left\{F_{\bar{x}} \mid \bar{x} \in \prod_{i=1}^{t}\{0,1\}^{k^{2}}\right\}$ is a $(3 / 4+\epsilon)$-approximate MaxIS family of lower bound graphs.

Lemma 3 is a corollary of Claims 6 and 7.
СцаIM 6. For any $g_{\bar{x}} \in\left\{F_{\bar{x}} \mid \bar{x} \in \prod_{i=1}^{t}\{0,1\}^{k^{2}}\right\}$, if there is a pair $\left(m_{1}, m_{2}\right) \in[k] \times[k]$ for which it holds that $x_{\left(m_{1}, m_{2}\right)}^{1}=x_{\left(m_{1}, m_{2}\right)}^{2}=$ $\cdots=x_{\left(m_{1}, m_{2}\right)}^{t}=1$, then $g_{\bar{x}}$ contains an independent set of weight at least $4 t \ell+2 \alpha t$.

Proof. Consider the following set of nodes.

$$
I=\bigcup_{i=1}^{t}\left\{v_{m_{1}}^{(i, 1)}\right\} \cup \operatorname{Cod} e_{m_{1}}^{(i, 1)} \cup\left\{v_{m_{2}}^{(i, 2)}\right\} \cup \operatorname{Cod} e_{m_{2}}^{(i, 2)}
$$

First, by Property 1, it holds that both $\bigcup_{i=1}^{t}\left\{v_{m_{1}}^{(i, 1)}\right\} \cup \operatorname{Code} e_{m_{1}}^{(i, 1)}$ and $\bigcup_{i=1}^{t}\left\{v_{m_{2}}^{(i, 2)}\right\} \cup \operatorname{Code} e_{m_{2}}^{(i, 2)}$ are independent sets. Furthermore, the only possible edges between $\bigcup_{i=1}^{t}\left\{v_{m_{1}}^{(i, 1)}\right\} \cup \operatorname{Code} e_{m_{1}}^{(i, 1)}$ and $\bigcup_{i=1}^{t}\left\{v_{m_{2}}^{(i, 2)}\right\} \cup$ $\operatorname{Code}_{m_{2}}^{(i, 2)}$ are the ones in $\left\{\left\{v_{m_{1}}^{(i, 1)}, v_{m_{2}}^{(i, 2)}\right\} \mid i \in[t]\right\}$. But since $x_{\left(m_{1}, m_{2}\right)}^{1}=x_{\left(m_{1}, m_{2}\right)}^{2}=\cdots=x_{\left(m_{1}, m_{2}\right)}^{t}=1$, none of the edges in $\left\{\left\{v_{m_{1}}^{(i, 1)}, v_{m_{2}}^{(i, 2)}\right\} \mid i \in[t]\right\}$ exists in the graph $F_{\bar{x}}$. The weight of $I$ is $\left|\bigcup_{i=1}^{t} w\left(\left\{v_{m_{1}}^{(i, 1)}, v_{m_{2}}^{(i, 2)}\right\}\right)\right|+\left|\bigcup_{i=1}^{t} w\left(\operatorname{Code}_{m_{1}}^{(i, 1)} \cup \operatorname{Code}_{m_{2}}^{(i, 2)}\right)\right|=$ $2 t \ell+2 t(\ell+\alpha)=t(4 \ell+\alpha)$, as desired.

Claim 7. For any

$$
g_{\bar{x}} \in\left\{G_{\bar{x}} \mid \bar{x} \in \prod_{i=1}^{t}\{0,1\}^{k^{2}}\right\}
$$

, if the strings $x^{1}, x^{2}, \cdots, x^{t}$ are pairwise disjoint, then the weight of any independent set in $g_{\bar{x}}$ is at most $3(t+1) \ell+3 \alpha t^{3}$.

Due to space limitations, the proof of Claim 7 can be found in the full version of the paper [12].

Proof of Lemma 3. Claims 6 and 7 imply that

$$
\left\{G_{\bar{x}}=\left(V, E_{\bar{x}}, w_{\bar{x}}\right) \mid \bar{x} \in \prod_{i=1}^{t}\{0,1\}^{k^{2}}\right\}
$$

is a family of lower bound graphs with respect to the pairwise disjointness function and the graph predicate that distinguishes between graphs of maximum independent set at least $4 t \ell+2 \alpha t$ and graphs of maximum independent set at most $3(t+1) \ell+3 \alpha t^{3}$.

Recall that $\ell=\log k-\log k / \log \log k, \alpha=\log k / \log \log k$. Which implies that the graph predicate distinguishes between independent sets of weight at least $4 t(\log k-\log k / \log \log k)+2 t \log k / \log \log k=$ $4 t \log k-2 t \log k / \log \log k \geq 4(t-1) \log k$ and independent sets of weight at most $3(t+1)(\log k-\log k / \log \log k)+3 t^{3}(\log k / \log \log k) \leq$ $3(t+2) \log k$, for any constant $t$ and $k \gg t$. Hence, for any constant $\epsilon>0$, we choose $t=(3 / 4 \epsilon)-1$ (or the first integer larger than $t=(3 / 4 \epsilon)-1$, if it is not an integer). This implies that for any constant $0<\epsilon \leq 1 / 4$, there is a constant $t$ for which $\left\{G_{\bar{x}}=\left(V, E_{\bar{x}}, w_{\bar{x}}\right) \mid \bar{x} \in \prod_{i=1}^{t}\{0,1\}^{k^{2}}\right\}$ is a $(3 / 4+\epsilon)$-approximate MaxIS family of graphs.

Proof of Theorem 2. Observe that $k=\Theta(t n)=\Theta(n)$, where $n=|V|$. Furthermore, by Lemma $3,\left\{G_{\bar{x}}=\left(V, E_{\bar{x}}, w_{\bar{x}}\right)\right\}$ is a $(3 / 4+\epsilon)$ approximate MaxIS family of graphs, where the partition of the set of nodes that is needed for Definition 4 is $V=\bigcup_{i=1}^{t} V^{i}$. Hence, 
by Corollary 1 , the fact that the length of the strings is $k^{2}=\Theta\left(n^{2}\right)$, and the fact that $\left|\operatorname{cut}\left(G_{\bar{x}}\right)\right|=\Theta\left(t^{2} \log ^{2} k\right)=\Theta\left(\log ^{2} k\right)$ (there are $\Theta\left(t^{2}\right)$ cuts between pairs of players, each of them contains $\theta\left((\ell+\alpha)^{2}\right)=\Theta\left(\log ^{2} k\right)$ edges $)$, any algorithm for finding a $(3 / 4+\epsilon)-$ approximation to maximum independent set in the CONGEST model with success probability at least $2 / 3$ requires $\Omega\left(k^{2} /(t \log t\right.$. $\left.\left.\left|\operatorname{cut}\left(G_{\bar{x}}\right)\right| \log |V|\right)\right)=\Omega\left(n^{2} /\left(t \log t \cdot \log ^{3} n\right)=\Omega\left(n^{2} / \log ^{3} n\right)\right.$ rounds.

Acknowledgments. We would like to thank Ami Paz for valuable discussions and useful comments. Yuval Efron was supported by the European Union's Horizon 2020 Research And Innovation Programme under grant agreement no. 755839, and also supported in part by the Binational Science Foundation (grant 2015803). Ofer Grossman was supported by a Fannie and John Hertz Foundation Fellowship, and an NSF GRFP fellowship. Finally, we thank the Simons institute for the generous hosting of the authors while performing this work.

\section{REFERENCES}

[1] Amir Abboud, Keren Censor-Hillel, and Seri Khoury. 2016. Near-Linear Lower Bounds for Distributed Distance Computations, Even in Sparse Networks. In 30th International Symposium on Distributed Computing, DISC. 29-42.

[2] Amir Abboud, Keren Censor-Hillel, Seri Khoury, and Christoph Lenzen. 2017. Fooling Views: A New Lower Bound Technique for Distributed Computations under Congestion. CoRR abs/1711.01623 (2017). arXiv:1711.01623 http://arxiv org/abs/1711.01623

[3] Sanjeev Arora and Boaz Barak. 2009. Computational Complexity - A Modern Approach. Cambridge University Press. http://www.cambridge.org/catalogue/ catalogue. asp?isbn=9780521424264

[4] Nir Bachrach, Keren Censor-Hillel, Michal Dory, Yuval Efron, Dean Leitersdorf, and Ami Paz. 2019. Hardness of Distributed Optimization. In Proceedings of the 2019 ACM Symposium on Principles of Distributed Computing, PODC 2019, Toronto, ON, Canada, fuly 29 - August 2, 2019. 238-247.

[5] Reuven Bar-Yehuda, Keren Censor-Hillel, Mohsen Ghaffari, and Gregory Schwartzman. 2017. Distributed Approximation of Maximum Independent Set and Maximum Matching. In Proceedings of the ACM Symposium on Principles of Distributed Computing, PODC. 165-174.

[6] Keren Censor-Hillel and Michal Dory. 2018. Distributed Spanner Approximation In Proceedings of the 2018 ACM Symposium on Principles of Distributed Computing, PODC 2018, Egham, United Kingdom, Fuly 23-27, 2018. ACM, 139-148.

[7] Keren Censor-Hillel, Telikepalli Kavitha, Ami Paz, and Amir Yehudayoff. 2016. Distributed Construction of Purely Additive Spanners. In Proceedings of the 30th International Symposium on Distributed Computing, DISC. 129-142.

[8] Keren Censor-Hillel, Seri Khoury, and Ami Paz. 2017. Quadratic and NearQuadratic Lower Bounds for the CONGEST Model. In 31st International Symposium on Distributed Computing, DISC 2017, October 16-20, 2017, Vienna, Austria 10:1-10:16.

[9] Amit Chakrabarti, Subhash Khot, and Xiaodong Sun. 2003. Near-Optimal Lower Bounds on the Multi-Party Communication Complexity of Set Disjointness. In 18th Annual IEEE Conference on Computational Complexity (Complexity 2003), 7-10 July 2003, Aarhus, Denmark. 107-117.

[10] Artur Czumaj and Christian Konrad. 2018. Detecting Cliques in CONGEST Networks. In 32nd International Symposium on Distributed Computing, DISC 2018, New Orleans, LA, USA, October 15-19, 2018 (LIPIcs), Vol. 121. Schloss Dagstuhl Leibniz-Zentrum fuer Informatik, 16:1-16:15.

[11] Andrew Drucker, Fabian Kuhn, and Rotem Oshman. 2014. On the power of the congested clique model. In Proceedings of the 33rd ACM Symposium on Principles of Distributed Computing, PODC. 367-376.

[12] Yuval Efron, Ofer Grossman, and Seri Khoury. 2020. Beyond Alice and Bob: Improved Inapproximability for Maximum Independent Set in CONGEST. CoRR abs/2003.07427 (2020). arXiv:2003.07427 https://arxiv.org/abs/2003.07427

[13] Michael Elkin. 2006. An Unconditional Lower Bound on the Time-Approximation Trade-off for the Distributed Minimum Spanning Tree Problem. SIAM 7. Comput. 36, 2 (2006), 433-456.

[14] Orr Fischer, Tzlil Gonen, Fabian Kuhn, and Rotem Oshman. 2018. Possibilities and Impossibilities for Distributed Subgraph Detection. In Proceedings of the 30th on Symposium on Parallelism in Algorithms and Architectures, SPAA 2018, Vienna, Austria, fuly 16-18, 2018. ACM, 153-162.
[15] Silvio Frischknecht, Stephan Holzer, and Roger Wattenhofer. 2012. Networks cannot compute their diameter in sublinear time. In Proceedings of the ACM-SIAM Symposium on Discrete Algorithms, SODA. 1150-1162.

[16] Mohsen Ghaffari and Fabian Kuhn. 2013. Distributed Minimum Cut Approximation. In Distributed Computing - 27th International Symposium, DISC 2013, Jerusalem, Israel, October 14-18, 2013. Proceedings (Lecture Notes in Computer Science), Vol. 8205. Springer, 1-15.

[17] Tzlil Gonen and Rotem Oshman. 2017. Lower Bounds for Subgraph Detection in the CONGEST Model. In 21st International Conference on Principles of Distributed Systems, OPODIS 2017, Lisbon, Portugal, December 18-20, 2017 (LIPIcs), Vol. 95. Schloss Dagstuhl - Leibniz-Zentrum fuer Informatik, 6:1-6:16.

[18] Stephan Holzer and Nathan Pinsker. 2015. Approximation of Distances and Shortest Paths in the Broadcast Congest Clique. In Proceedings of the 19th International Conference on Principles of Distributed Systems, OPODIS. 6:1-6:16.

[19] Ken-ichi Kawarabayashi, Seri Khoury, Aaron Schild, and Gregory Schwartzman. 2019. Improved Distributed Approximation to Maximum Independent Set. CoRR abs/1906.11524 (2019). arXiv:1906.11524 http://arxiv.org/abs/1906.11524

[20] Eyal Kushilevitz and Noam Nisan. 1997. Communication Complexity. Cambridge University Press, New York, NY, USA.

[21] Danupon Nanongkai, Atish Das Sarma, and Gopal Pandurangan. 2011. A tight unconditional lower bound on distributed randomwalk computation. In Proceedings of the 30th Annual ACM Symposium on Principles of Distributed Computing, PODC 2011, San fose, CA, USA, fune 6-8, 2011. ACM, 257-266.

[22] David Peleg. 2000. Distributed Computing: A Locality-Sensitive Approach. Society for Industrial and Applied Mathematics, Philadelphia, PA, USA.

[23] David Peleg and Vitaly Rubinovich. 1999. A Near-Tight Lower Bound on the Time Complexity of Distributed MST Construction. In Proceedings of the 40th Annual Symposium on Foundations of Computer Science, FOCS. 253-261.

[24] David Peleg and Vitaly Rubinovich. 2000. A Near-Tight Lower Bound on the Time Complexity of Distributed Minimum-Weight Spanning Tree Construction. SIAM 7. Comput. 30, 5 (2000), 1427-1442.

[25] Jeff M. Phillips, Elad Verbin, and Qin Zhang. 2012. Lower bounds for numberin-hand multiparty communication complexity, made easy. In Proceedings of the Twenty-Third Annual ACM-SIAM Symposium on Discrete Algorithms, SODA 2012, Kyoto, Japan, January 17-19, 2012. 486-501.

[26] Alexander A. Razborov. 1992. On the Distributional Complexity of Disjointness. Theor. Comput. Sci. 106, 2 (1992), 385-390.

[27] Atish Das Sarma, Stephan Holzer, Liah Kor, Amos Korman, Danupon Nanongkai, Gopal Pandurangan, David Peleg, and Roger Wattenhofer. 2011. Distributed verification and hardness of distributed approximation. In Proceedings of the 43rd ACM Symposium on Theory of Computing, STOC 2011, San fose, CA, USA, 6-8 fune 2011. ACM, 363-372.

[28] Atish Das Sarma, Stephan Holzer, Liah Kor, Amos Korman, Danupon Nanongkai, Gopal Pandurangan, David Peleg, and Roger Wattenhofer. 2012. Distributed Verification and Hardness of Distributed Approximation. SIAM 7. Comput. 41, 5 (2012), 1235-1265. 